\title{
Nanocrystals in their prime
}

\author{
Three decades after the first fundamental studies on semiconductor nanocrystals, the materials can be \\ found in a range of commercial devices.
}

In semiconductor nanocrystals, electron motion is confined in all three dimensions. This confinement leads to energy quantization and an optical absorption spectrum with discrete lines, as in the case of atoms. By varying the size or composition of the nanocrystals, the optical emission can be tuned over a wide range of wavelengths from the far-infrared to ultraviolet - and, as a result, the materials are of significant use in a range of applications.

Research into semiconductor nanocrystals, which are also known as colloidal quantum dots, began in the early 1980s. Alexei Ekimov, working at the Vavilov State Optical Institute, was studying the colour formation of semiconductor-doped glasses. Whilst experimenting with glasses activated by $\mathrm{CdS}, \mathrm{CdSe}, \mathrm{CuCl}$ and $\mathrm{CuBr}$ he discovered the presence of nanosized crystals of these semiconductors in the glasses and that these nanocrystals could absorb light at much lower wavelengths than expected. Ekimov subsequently sought help from the theoretician Alexander Efros working at the Ioffe Institute, and the two researchers eventually demonstrated that the optical properties of these semiconductor crystals were dominated by their size ${ }^{1}$.

Around the same time, Louis Brus at Bell Labs was working on CdS colloids. Through a fine adjustment of growth conditions, he was able to control the size of these colloids and created nanocrystals that had diameters as small as $4.5 \mathrm{~nm}$. He found that the absorption spectra of these nanocrystals exhibited a substantial blueshift compared with bulk CdS, which was consistent with a quantum confinement of electrons and holes ${ }^{2}$.

Three decades later, our understanding and control of the growth and properties of nanocrystals has reached an exceptional level. Advances in synthesis have provided ways to control the shape - and thus the electronic and optical properties. In 1993, for example, a recipe for the synthesis of nearly monodisperse spherical nanocrystals was introduced $^{3}$. A few years later the formation of nanorods was reported ${ }^{4}$. Of particular interest are semiconductor nanoplatelets ${ }^{5}$, which are a few tens of nanometres wide but only a few atomic layers thick. Nanoplatelets are alternatives to the recently developed dichalcogenide foils such as $\mathrm{MoS}_{2}$ and $\mathrm{WSe}_{2}$,

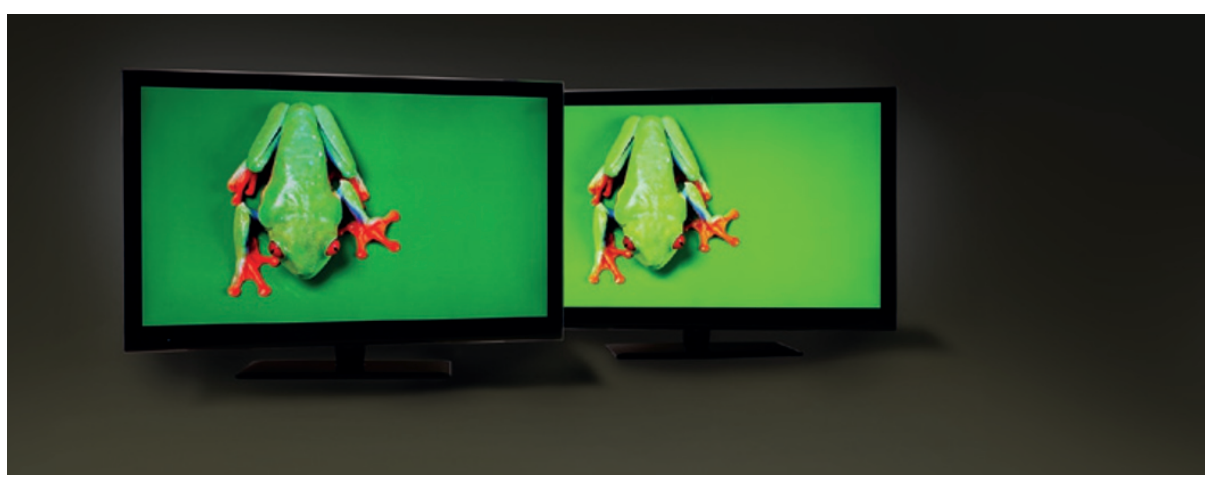

Figure 1 | Comparison between a liquid-crystal display television screen using a Quantum Dot Enhancement Film (left) and a standard one (right). Photo courtesy of Nanosys/Thom Sanborn.

but with the advantage that they can be synthesized and processed in solution.

Research into nanocrystals has recently been translated into the production of commercial devices. In May 2011, the company Nanosys announced a product called the Quantum Dot Enhancement Film This is a thin layer of quantum dots that convert the blue light of a GaN light-emitting diode into bright colours and can be used in liquid-crystal displays (Fig. 1). Around the same time, Samsung announced the creation of a 4-inch quantum-dot-based colour display, with higher definition and lower power consumption than commercial liquidcrystal displays; a development that they suggest will lead to the commercialization of full-screen quantum-dot displays in the next few years. Furthermore, the Amazon Kindle uses quantum dot technology for enhanced definition, and Sony uses nanocrystals made by the company QD Vision in some models of its televisions.

Several companies are also pursuing white light-emitting diodes based on nanocrystals, and research effort is concentrated on trying to improve the performance of lasers and solar cells based on such nanocrystals. For solar cells, the solar-power conversion efficiency is still relatively low, but it has been raised from 1\% in 2005 to $8.5 \%$ in $2013^{7}$. Improvements in charge mobility and extraction will probably improve their efficiency further in the next few years.

Advances in the optical properties of semiconductor nanocrystals have also enabled them - when encapsulated in polymeric shells - to be used in bioimaging and diagnostic applications ${ }^{8}$. These materials offer a number of advantages over the commonly used organic dyes, including brightness and resistance to photobleaching and blinking. There is, however, room for substantial improvement for their use in biological applications. For example, the polymeric shell significantly increases the size of the nanocrystals and makes it more difficult for them to be incorporated into tissues. The nanocrystals also tend to bind non-specifically, which makes targeting particular biomolecules a challenge. Finally, the use of heavy metals such as cadmium in the nanocrystals raises concerns about toxicity and has limited their use to in vitro studies so far.

The success of the first 30 years of semiconductor nanocrystals will be celebrated later this month at a conference in Paris, featuring keynote talks from Ekimov, Efros and Brus ${ }^{9}$. In three decades, the materials have found their way into a number of commercial devices and applications, and it seems likely that more will follow.

\footnotetext{
References

1. Ekimov, A. I., Efros, Al. L. \& Onushchenko, A. A. Solid State Commun. 56, 921-924 (1985).

2. Brus, L. E. J. Chem. Phys. 80, 4403-4409 (1984).

3. Murray, C. B., Norris, D. J. \& Bawendi, M. G. J. Am. Chem. Soc 115, 8706-8715 (1993).

4. Peng, X. G. et al. Nature 404, 59-61 (2000).

5. Ithurria, S. \& Dubertret, B. J. Am. Chem. Soc 130, 16504-16505 (2008).

6. Nanosys; available via http://go.nature.com/873Svb

7. Lan, X., Masala, S. \& Sargent, E. H. Nature Mater. 13, 233-240 (2013)

8. Kairdolf, A. B. et al. Annu. Rev. Anal. Chem. 6, 143-162 (2013)

9. http://www.30-years-qds.com/
} 